\title{
ATM Switch Implementation for NGN using DWDM
}

\author{
J. Anjani Ramya \\ PG Student \\ GVP College of Engineering $(A)$ \\ Visakhapatnam
}

\author{
P. Pavani \\ Assistant professor \\ GVP College of Engineering (A) \\ Visakhapatnam
}

\author{
G. Tirumala Rao \\ Professor \\ GVP College of Engineering(A) \\ Visakhapatnam
}

\begin{abstract}
In global communications the demand for higher bandwidth is increasing phenomenally due to the spread of Internet in particular and other domain applications in general. Legacy networks are existing networks which are in implementation. Continuity can be achieved through Optical backbone networks only, even though other media, such as satellite are available in public switched telephone networks, the interface between client and network is an optical Asynchronous Transfer Mode (ATM) switch.
\end{abstract}

Wavelength division multiplexing provides efficient bandwidth utilization. Dense wavelength division multiplexing uses multiplexers at the transmitter at different wavelengths. Availability of contiguous wavelength in the route should be considered for optimum utilization of routing algorithm. In terms of routing packets in optical domain, QOS parameters that need to be considered for optimization are Packet Size, Throughput, Traffic Density, Data Rates and BER.

This paper deals with understanding the parameters for various algorithms for duplex/bidirectional multiple packet transmission through asynchronous transfer mode (ATM) protocol. Here ATM acts as a switch for routing algorithm. Fixed Size ATM Packets are transmitted to optimize throughput in the network. NETLOGO software is used for simulation and analysis.

\section{Keywords}

ATM, DWDM, QOS, NETLOGO

\section{INTRODUCTION}

All-optical network technologies employing wavelength division multiplexing (WDM) is playing a major role in constructing large bandwidth networks. Since such networks require not only transmission line capability enhancement, WDM should be utilized in combination with wavelength routing. The function of routing must be implemented for reaching high connectivity and flexibility of optical network.

The need is for higher bandwidths handling of the aggregate future traffic load in Next Generation Networks (NGN) [1]. Optical networks can only support higher bandwidths. Bandwidth utilization and QoS can be improved with the implementation of the combination of Dense Wave Division Multiplexing (DWDM) and Asynchronous Transfer Mode (ATM).

Dense Wavelength Division Multiplexing (DWDM) Systems uses a number of multiplexers at the transmitter side at different wavelengths onto a single fiber [2].
ATM networks have the advantages of integrated switching and transport capability and a universal interface. All the network resources can be efficiently shared by a variety of services. Another advantage is the network flexibility provided by the ATM technique. This flexibility includes the dynamic network reconfiguration capability which enhances network performance. The integrated transport capability and the flexibility of the network are the key issues for future transport networks which should keep evolving to meet unknown user demands.

The global network consists of more than one Autonomous Systems (ASes) which are inter-linked topologically by using Gateways. These autonomous Systems are known as Domains. In optical network routing, a light path is established across multiple domains by determining an optimum route.

An Optical or light Path is the optical connection [3] maintained between a source node and destination nodes. The problem of finding a route for the optical path and assigning a wavelength to the each link is referred to as Routing and wavelength Assignment problem. Assigning a wavelength and transmitting a packet with VPI through its destination. A virtual path is a bundle of virtual channels, all of which are switched transparently across the ATM network based on the common VPI.

This paper is organized as follows. Section II gives overview on ATM Routing and Wavelength Assignment. Section III explains the operation of ATM protocol and Wavelength assignment. Section IV describes path computation algorithm. Section V presents Simulation, results and analysis. Section VI presents Conclusion.

\section{ATM ROUTING WAVELENGTH ASSIGNMENT}

The optical connection that is maintained between a source node and destination node is known as an optical path or light path. The problem of finding a route for a light path and assigning a wavelength to the light path is referred to as the routing and wavelength assignment (RWA) problem.

Here ATM acts as a switch. End system is also ATM switch. Fixed size data is transmitted bidirectional. To establish the light paths in all optical DWDM networks [4], a signaling protocol is required to request and setup the light path through the optical network. Various steps involved in RWA are shown in the Fig 1. 


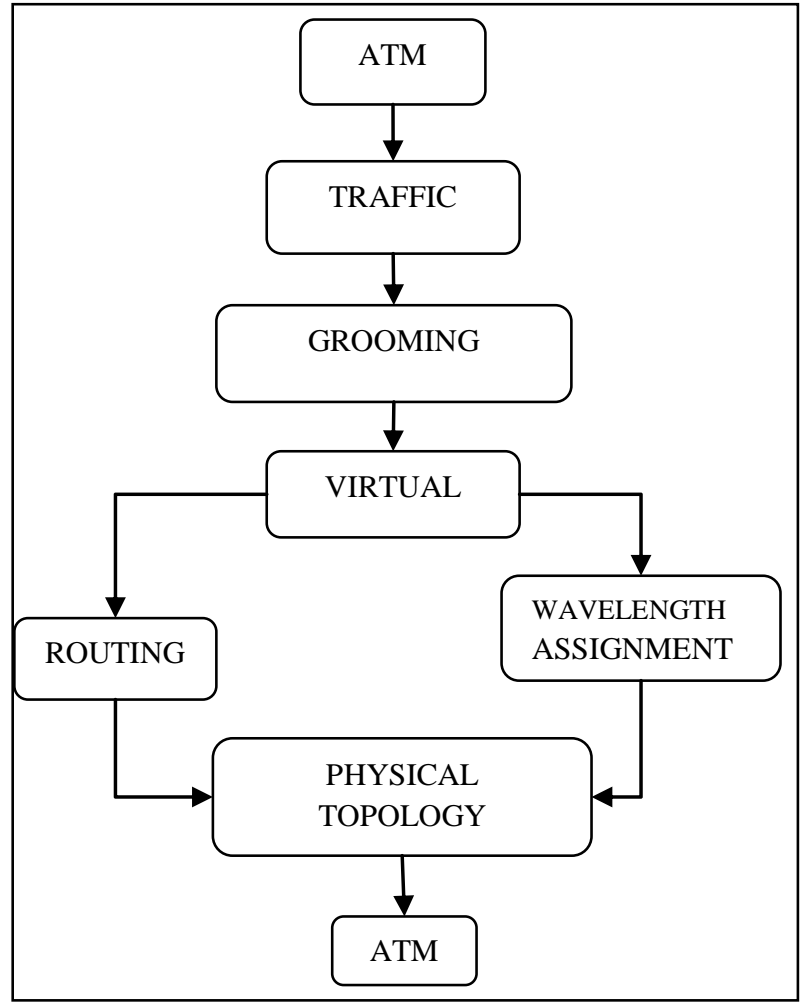

Fig 1: Routing and Wavelength Assignment

The incoming traffic is ATM 53 bytes size packets. These packets undergo grooming, [5] a technique to combine lower speed traffic onto available high speed wavelengths in order to meet network design goals. In a network using both timedivision multiplexing (TDM) and wavelength-division multiplexing (WDM), two flows which are destined for a common node can be placed on the same wavelength, allowing them to be dropped by a single optical add-drop multiplexer.

These two steps are used to find an optimal route from source to destination. This route is linked to the physical network. All connection requests are known in advance in this method of light path establishment and they do not change. Once optical paths are setup they are not released. However, Wavelength continuity constraint is a problem in establishing light paths.

\subsection{Dynamic Lightpath Establishment (DLE)}

In this method all connection requests arrive dynamically [6]. The objective of RWA problem is to setup a lightpath for each connection request such that the blocking probability for future connection requests is minimized and also to minimize network cost.

\subsection{Routing}

Routing is a process to bridge the packet of data from source to destination using dedicated devices like routers, gateways, switches. Routing involves two basic activities: determining the optimal path and transporting packets through a network. The routing is performed on two levels: Inter domain and Intra domain [7].

Two types of routing protocols are used in Autonomous Systems: Interior Gateway Protocol (IGP) and Exterior Gateway Protocol (EGP). IGP is used to distribute routing within a single Autonomous System. EGP is used to distribute the reachability information of the external to the AS (i.e. neighboring AS's). In both the domains each packet is being traversed to desired destination by assigning wavelength to each link.

\section{WAVELENGTH ASSIGNMENT}

After selecting a route for the connection request, an appropriate wavelength needs to be assigned to setup a light path from source to destination nodes. Fig 2 shows the wave length Assignment [8].

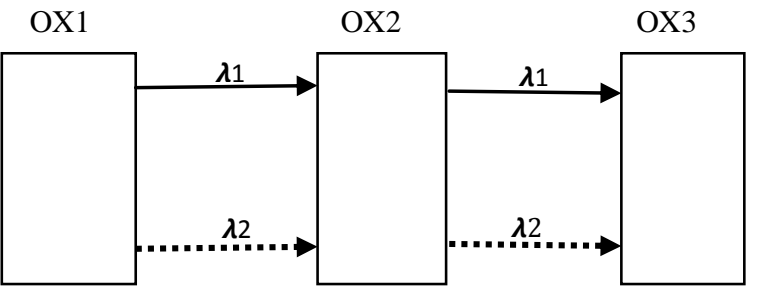

Fig 2: Wavelength Assignment

Consider three Optical Cross Connector (OXC1, OXC2 and OXC3) in a network [9]. If a packet of information has to be transferred from $\mathrm{OXC1}$ to $\mathrm{OXC} 3$, a light path has to be established from Source (OXC1) to Destination (OXC3), that light path has to be assigned with a wavelength available in the entire path from source to destination. In the figure $2, \lambda_{2}$ wavelength is available in both the links OXC1 to OXC2 and $\mathrm{OXC} 2$ to OXC3. Therefore the light path can be assigned with the wavelength $\lambda_{2}$ which satisfies the wavelength continuity. The Wavelength Continuity Constraint situation is shown in Fig 3.

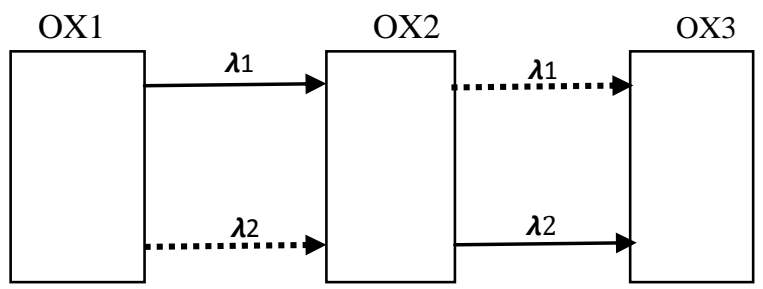

Fig 3: Wavelength Continuity Constraint

Suppose if a light path between $\mathrm{OXC} 1$ and $\mathrm{OXC} 2$ established with the wavelength $\lambda_{1}$ and another light path between OXC2 and OXC3 established with the wavelength $\lambda_{2}$. Now we assume that we need to establish a light path from $\mathrm{OXC} 1$ to OXC3. This is not possible even we have free wavelengths on each hop from OXC1 to OXC3. This condition is called Wavelength Continuity Constraint.

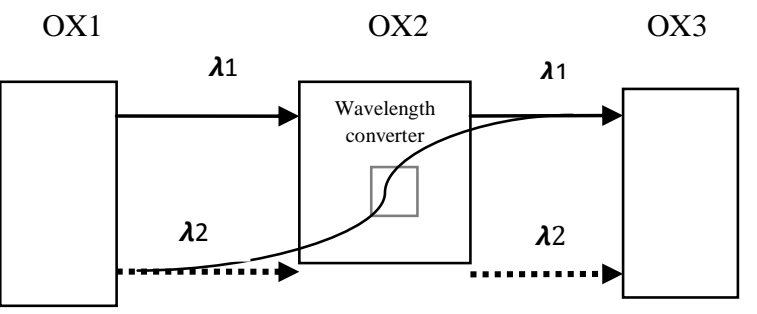

Fig: 4 Wavelength Converters

The wavelength continuity Constraint can be relaxed with the usage of Wavelength Converters. Above Fig 4 shows the wave length conversion. The Wavelength Converter converts the input signal wavelength to required wavelength. The usage of the wavelength converters can reduce the blocking probability. 


\section{PATH COMPUTATION ALGORITHM}

Path computation algorithm is summarized as follows

1. A network of nodes has to be Setup.

2. A Source Node and a Destination are identified.

3. For the selected source and destination, packet is inserted on the source node.

4. Wavelength continuity has to be checked for each node in the path.

5. Wavelength continuity is checked for the next node and transmits the packet with VPI.

6. If not VPI node checks with cost metric and transmit the packet .If the wavelength continuity is not achieved then it checks for new wavelengths in list.

7. The alternate shortest path is generated by not considering (virtually disabling) the node next to the node where wavelength discontinuity is arrived.

8. The node where the discontinuity arrived is considered as source node keeping destination node unchanged.

9. Loop back to Step 3 until a path is generated with wavelength continuity.

10. Bidirectional packet transmission is done in multi domain environment.

The flow diagram of path computation is shown in Fig.5.

The paper analyzed routing of multi-domain optical network with Wavelength Continuity Constraint in the absence of Wavelength Converters using the NETLOGO simulation tool. NETLOGO software is used to simulate the network in this paper. NETLOGO is an agent based programmable modeling environment for Simulation of complex networks.

Shortest Path is calculated by considering distance as the metric with wavelength assignment and virtual light path establishment is done by considering wavelength continuity constraint for both Intra domain and Inter domain Networking.

\section{RESULTS}

A complex network of 33 nodes with three autonomous systems is considered for simulation. Shortest path is calculated for a given source node and destination node using ATM Protocol. These routers are responsible in interlinking different Autonomous Systems. Consider it as AS_1. The nodes $0,1,2 \ldots 15$ are internal routers for AS_1. The nodes $16,17 \ldots 22$ are internal routers for AS_2. Nodes 23, 24, .32 are internal routers for AS_3. By using ATM protocol shortest path and wavelength continuity are done for multi-packet transmission.

The Shortest Path from Node 11 to Node 22(located in different Autonomous systems) is generated and wavelength continuity constraint is also verified.

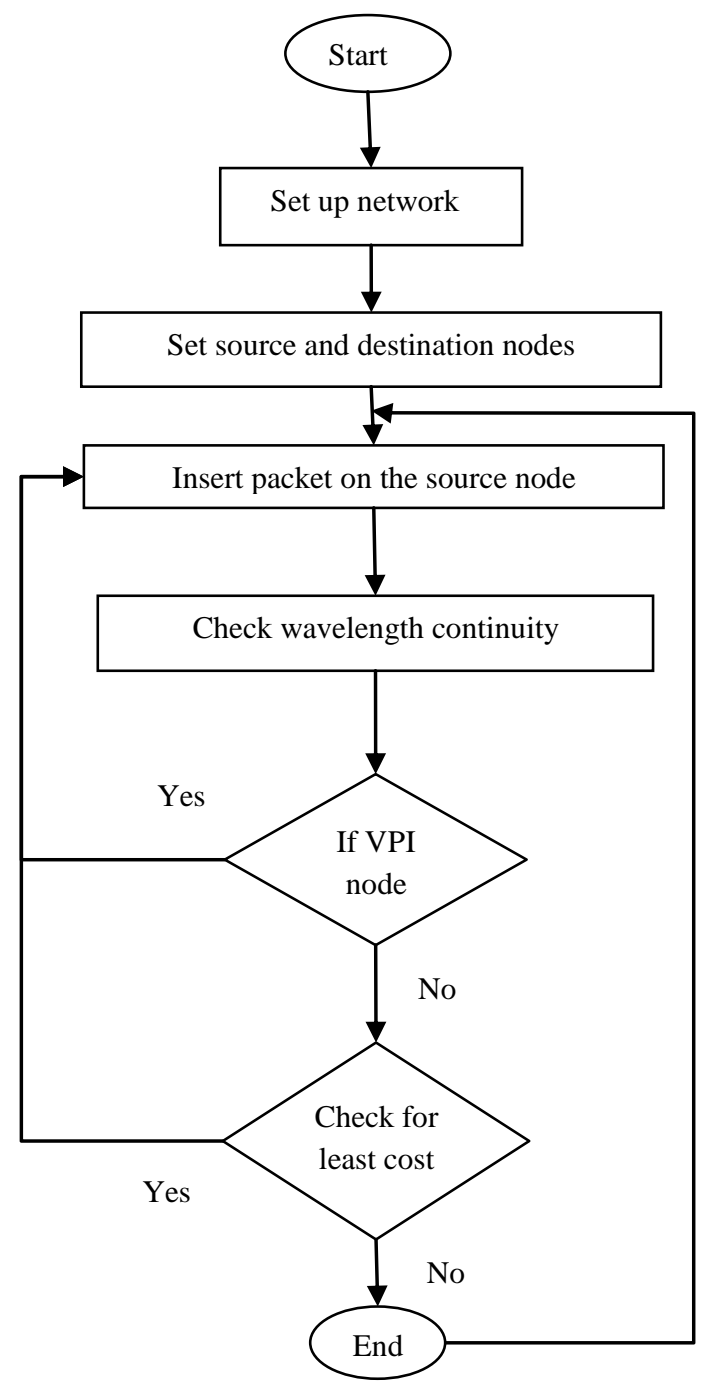

Fig 5: Flow diagram of Path Computation Algorithm

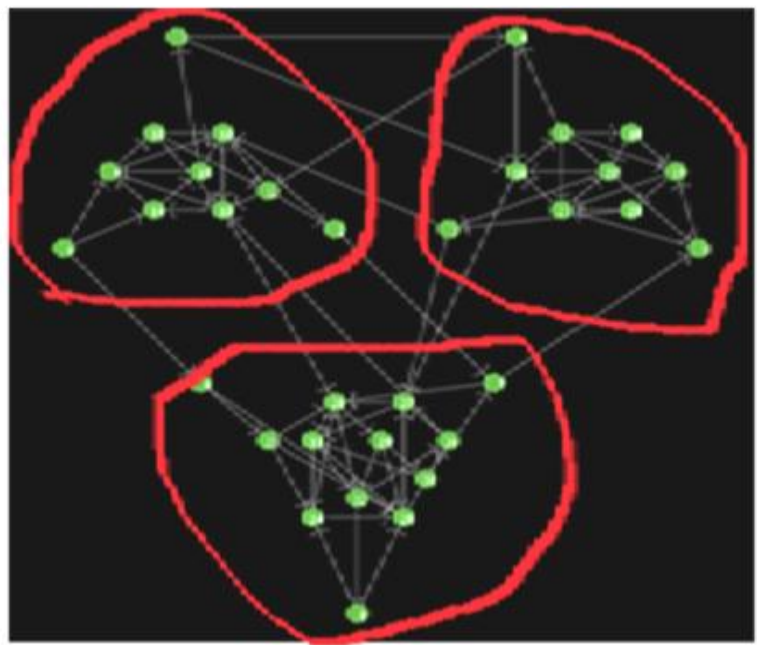

Fig 6: Multi domain network 
As shown in Fig 7, in inter domain routing for a given source node15 and destination node 21 , packet is transmitted by considering wavelength continuity constraint and packet is transmitted by considering virtual path identifier VPI towards desired destination.

Allotting VPI for each path as wavelength is constraint unlimited. The incoming wavelength is taken as outgoing wavelength with VPI.

Similarly light path from Node 15 to Node 22 (located in different autonomous Systems) is generated by allotting VPI for each path as wavelength constraint unlimited. Bidirectional packet is transmitted as shown in above Fig.8.In dynamic environment cost is taken as a metric for finding VPI node along with wavelength condition.

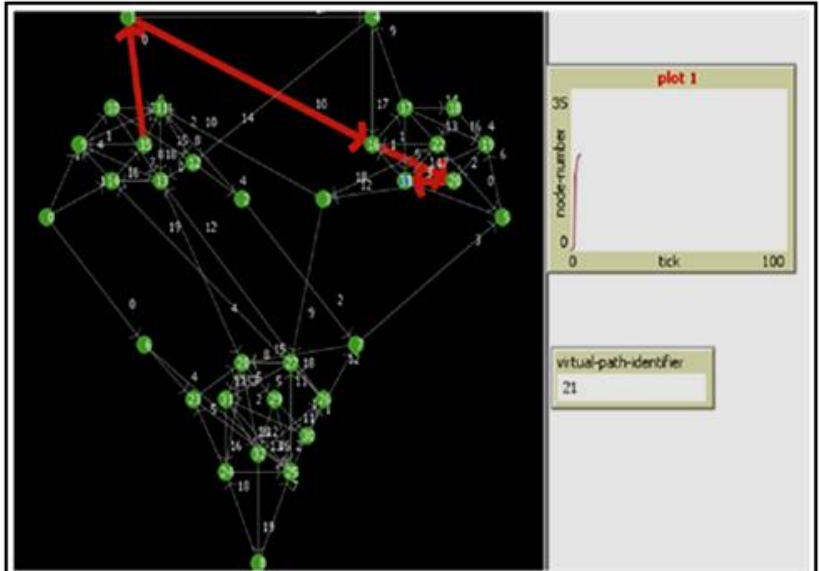

Fig 7: packet transmission in interdomain

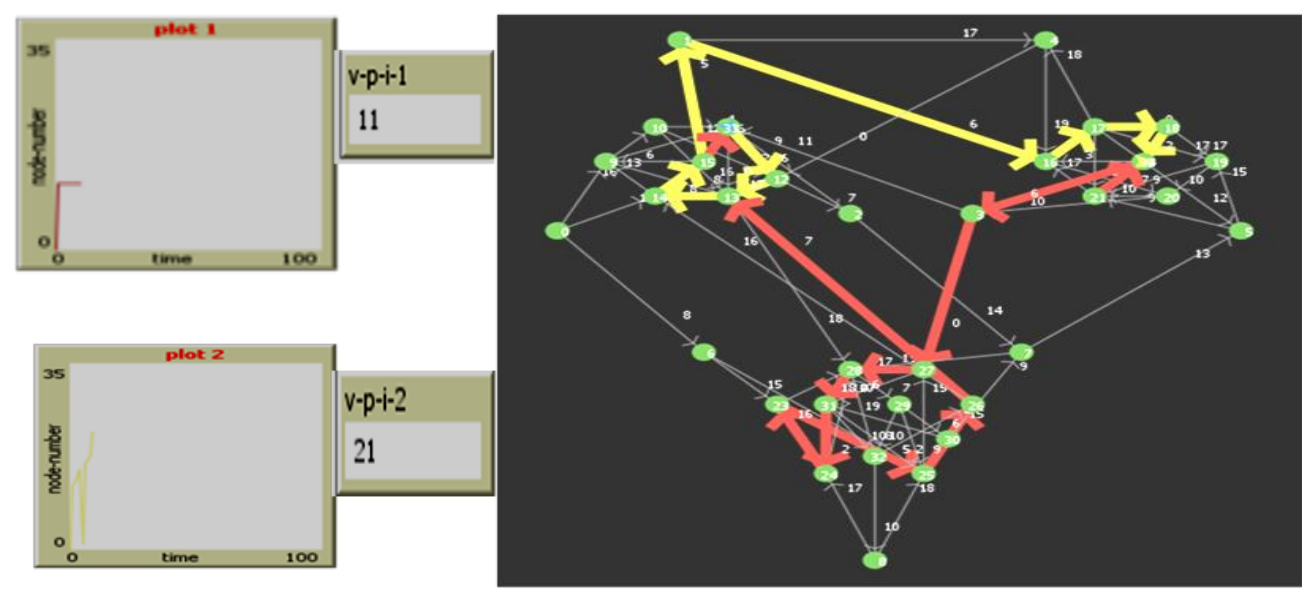

Fig 8: Bidirectional packet transmission

\section{CONCLUSION}

In this paper, Routing and Wavelength Assignment in MultiDomain Optical Network with Wavelength Continuity Constraint is analyzed. Random wavelength environment means the available wavelengths in a link are randomly considered. Wavelength Assignment for a packet is the main factor affecting the blocking probability. Path Computational Algorithm is developed for transmission of fixed size packets by implementing ATM protocol with a common wavelength, with which the packet is traversing. Due to WCC blocking probability arrives. In case WCC is not satisfied, and packets are blocked, path computation algorithm searches for wavelengths from the previous node.

In all the simulations, DWDM traffic flows which are simulated transfer data between domains. Study on opticalVPNs can be done which provide a virtual infrastructure for end-users to manage their own site-to site connections bandwidth allocation and circuit protection options within the $\mathrm{O}-\mathrm{VPN}$ domain which is the future scope of this paper.

\section{REFERENCES}

[1] ITU-T" NGN GSI Introduction" (http:// www . itu .int /en /ITU-T /gsi / ngn / Pages / introduction . aspx Updated :2010-09-22).

[2] Gerald P.Rayn, "Dense Wavelength Division Multiplexing": ATG's Communications \& Networking Technology Guide Series pp: 1-23, 1997.
[3] Tellabs, "Integrated Next -Generation WDM Transport", 2005.

[4] CISCO SYSTEMS, "Introduction to DWDM Technology".

[5] Tanmay De, Puneet Jain, Ajit Pal and Indranil Sengupta, "A Genetic algorithm Based Approach for Traffic Grooming, Routing and Wavelength Assignment in Optical WDM Mesh Network", 978-1-4244-38051/08@IEEE,2008.

[6] Ling Li and Arun K.Somani, "Dynamic Wavelength Routing Using Congestion and Neighborhood Information", Volume: 7, PP: 779 - 786, IEEE/ACM TRANSACTIONS ON NETWORKING, 1999.

[7] Xi Yang and Byrav Ramamurthy, "Inter- Domain Dynamic Routing in Multi-Layer Optical Transport Networks", Volume: 5, PP 2623 - 2627, GLOBECOM '03. IEEE Global Telecommunications Conference, 2003.

[8] P.Pavani, N.Balasubrahmanyam, G.TirumalaRao, "Routing in Multi Domain Environment Using IS-IS and BGP for QOS Improvement”, ACCT-2012, VOLUME-1, ISSUE-1, PAGE NO. 476-479, 2012

[9] K.Sampath Kumar,N.Balasubrahmanyam,G.Tirumala Rao, "Routing in Multi Domain Environment using OSPF \& BGP for QOS Improvement", ACCT-2012, Volume-1, Issue-1,Pageno:480-485,2012. 